Ultrafast spectral dynamics of dual-color-soliton intracavity collision in a mode-locked fiber laser

Yuan Wei, Bowen Li, Xiaoming Wei, Ying Yu, and Kenneth K. Y. Wong

Citation: Appl. Phys. Lett. 112, 081104 (2018); doi: 10.1063/1.5020821

View online: https://doi.org/10.1063/1.5020821

View Table of Contents: http://aip.scitation.org/toc/apl/112/8

Published by the American Institute of Physics 


\title{
Ultrafast spectral dynamics of dual-color-soliton intracavity collision in a mode-locked fiber laser
}

\author{
Yuan Wei, ${ }^{1, a)}$ Bowen $\mathrm{Li}^{1,}{ }^{1, a), b)}$ Xiaoming Wei, ${ }^{1,2}$ Ying Yu, ${ }^{1}$ and Kenneth K. Y. Wong ${ }^{1, b)}$ \\ ${ }^{1}$ Department of Electrical and Electronic Engineering, The University of Hong Kong, Pokfulam Road, \\ Hong Kong, China \\ ${ }^{2}$ Division of Engineering and Applied Science, California Institute of Technology, Pasadena, \\ California 91125, USA
}

(Received 27 December 2017; accepted 9 February 2018; published online 23 February 2018)

\begin{abstract}
The single-shot spectral dynamics of dual-color-soliton collisions inside a mode-locked laser is experimentally and numerically investigated. By using the all-optically dispersive Fourier transform, we spectrally unveil the collision-induced soliton self-reshaping process, which features dynamic spectral fringes over the soliton main lobe, and the rebuilding of Kelly sidebands with wavelength drifting. Meanwhile, the numerical simulations validate the experimental observation and provide additional insights into the physical mechanism of the collision-induced spectral dynamics from the temporal domain perspective. It is verified that the dynamic interference between the soliton and the dispersive waves is responsible for the observed collision-induced spectral evolution. These dynamic phenomena not only demonstrate the role of dispersive waves in the sophisticated soliton interaction inside the laser cavity, but also facilitate a deeper understanding of the soliton's inherent stability. Published by AIP Publishing. https://doi.org/10.1063/1.5020821
\end{abstract}

As the remarkable signature of many nonlinear optical systems, optical solitons, localized solitary waves stabilized by the delicate interplay between dispersive and nonlinear effects, have attracted numerous research attention. ${ }^{1,2}$ Thanks to their distinctive features, a wealth of sophisticated soliton dynamics in resonant cavities has been extensively explored, ${ }^{3-8}$ which includes rogue waves, ${ }^{9,10}$ soliton explosions, ${ }^{11-13}$ multiplesoliton generation, ${ }^{14}$ soliton molecules, ${ }^{15,16}$ and soliton rains. ${ }^{17}$ In addition to the single-color soliton, mode-locking can also be simultaneously achieved at multiple distinct spectral bands, ${ }^{18-23}$ which creates coexisting multi-color solitons. Optical frequency combs with slightly different comb spacing can therefore be conveniently obtained from a single laser cavity, which is a promising optical source for dual-comb spectroscopy. $^{21,24-28}$ On the other hand, the solitons with different group velocities owing to the chromatic dispersion will inevitably collide with each other from time to time. Such intracavity soliton interactions not only directly influence the performance of the dual-comb sources, but also incubate an intriguing regime of soliton dynamics, which however has never been fully unveiled owing to the insufficient frame rate of conventional optical spectrum analyzers (OSAs).

In this work, we report the roundtrip-resolved spectral dynamics of the dual-color-soliton intracavity collision in a nonlinear polarization rotation (NPR) mode-locked fiber laser by dispersive Fourier transform (DFT). ${ }^{29,30}$ The modelocked fiber laser under investigation simultaneously generates dual-color solitons of central wavelengths at $1532.2 \mathrm{~nm}$ and $1558.2 \mathrm{~nm}$, which we denote as blue and red solitons, respectively. Owing to their different group velocities, the blue and red solitons will inevitably collide with each other periodically as they circulate inside the cavity. Within a short

\footnotetext{
${ }^{a)}$ Y. Wei and B. Li contributed equally to this work.

b) Electronic addresses: bwli@eee.hku.hk and kywong@eee.hku.hk.
}

interacting time $(17.1 \mathrm{~ns})$, the collision significantly influences the physical characteristics of both solitons in both spectral and temporal domains. With the assistance of the DFT, the collision-induced spectral dynamics is round-trip resolved using a high-speed real-time oscilloscope at a spectral resolution of $0.09 \mathrm{~nm}$, which reveals the solitons' intriguing behavior during the post-collision self-reshaping process. Furthermore, numerical simulation is performed to validate our experimental spectral observation and provide additional insights into the physical mechanism from the time-domain perspective. We identify that collision-induced dispersivewave shedding and the interference between the soliton and the dispersive waves are responsible for the experimentally observed dual-color-soliton spectral dynamics. These unique dynamic phenomena not only represent a demonstration of soliton's inherent stability but will also inspire the exploration of analogous scenarios in other nonlinear systems. ${ }^{31}$

The laser under investigation in Fig. 1(a) is a passively mode-locked erbium-doped fiber (EDF) laser based on NPR. It consists of two in-line polarization controllers (PC), a 980/ $1550 \mathrm{~nm}$ wavelength-division multiplexer (WDM 1), a 90/10 beam splitting coupler for laser output, and a polarizationsensitive isolator (PS-ISO), a 2.5-m lowly-doped EDF and a 24.7-m standard single-mode fiber (SMF) with the total group-delay dispersion (GDD) of $-0.48 \mathrm{ps}^{2}$. Two PCs are placed before and after the SMF to manipulate the state of polarization (SOP) of lightwaves. Here, the PS-ISO, which functions as both an isolator and a polarizer, works with the PCs together to form an artificial saturable absorber. By well tuning the PCs and the pump power, the gain spectrum can be adjusted to obtain two identical gain peaks, which leads to the dual-color mode-locking regime.

The DFT module comprises a spool of a dispersioncompensating fiber (DCF) with a dispersion of $-2 \mathrm{~ns} / \mathrm{nm}$ at $1545 \mathrm{~nm}$, an erbium-doped fiber amplifier (EDFA) to 

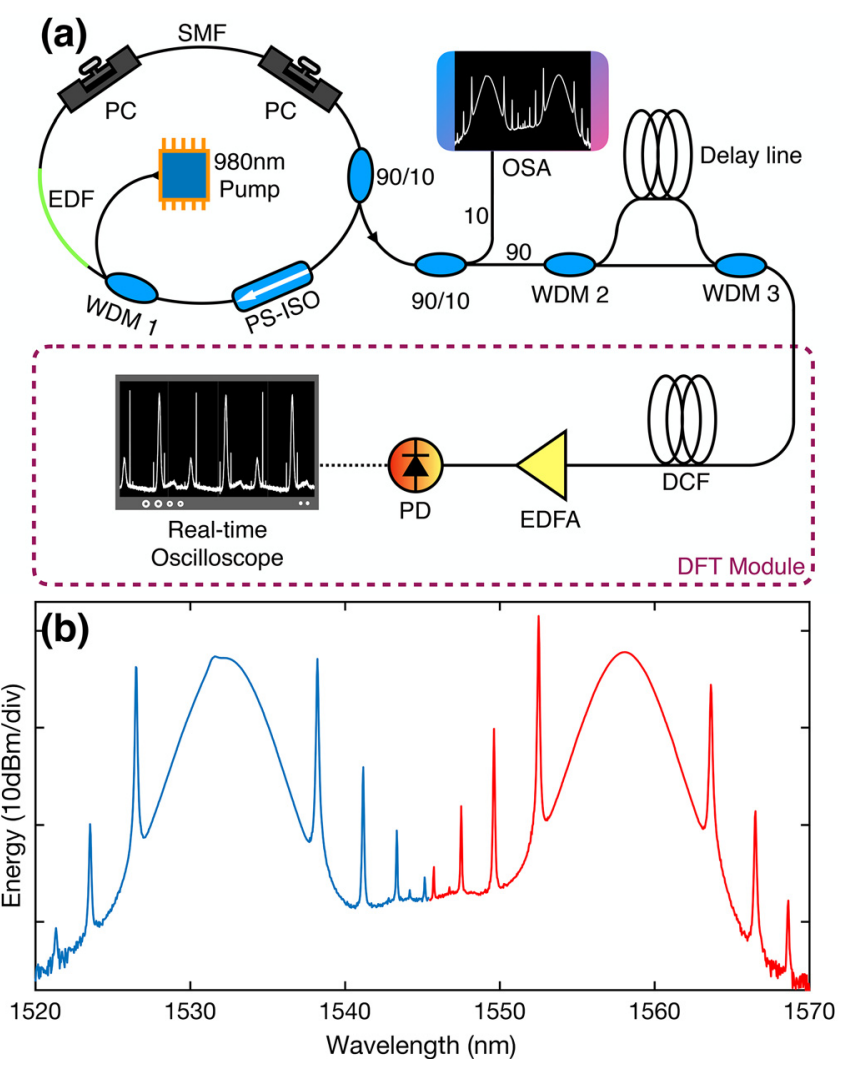

FIG. 1. (a) The schematic diagram of the experimental setup. (b) The OSA measured spectrum of dual-color soliton mode-locking. SMF, single-mode fiber; EDF, erbium-doped fiber; PC, polarization controller; WDM, wavelength-division multiplexer; PS-ISO, polarization-sensitive isolator; DCF, dispersion-compensating fiber; and EDFA, erbium-doped fiber amplifier.

compensate solitons' transmission loss induced by DCF, and a 20-GHz-bandwidth, 80-GS/s-sampling-rate real-time oscilloscope equipped with a $16-\mathrm{GHz}$ photodetector (PD) to conduct high-speed data acquisition, which renders the spectral resolution of $0.09 \mathrm{~nm}^{32}$ To prevent extra-cavity collision, WDM 2/3 (10-dB passband: $1524 \mathrm{~nm}-1546 \mathrm{~nm}$ ) is used to split/combine the blue and red solitons, and a piece of SMF delay line is inserted into the blue soliton branch. The ensemble spectrum measured by OSA in Fig. 1(b) verifies that the laser operates at a dual-color soliton mode-locking state. The periods of blue and red solitons were $133.16 \mathrm{~ns}$ and $133.17 \mathrm{~ns}$, respectively, which lead to a mutual collision every $1.8 \mathrm{~ms}$ inside the cavity. The final temporal waveform after the DFT module is recorded using the real-time oscilloscope and post-processed to reconstruct the round-trip resolved spectral evolution.

Figure 2(a) shows the reconstructed spectral evolution over 120 consecutive roundtrips. The initially stable soliton spectrum is obviously distorted after the collision (the $21 \mathrm{st}$ roundtrip). To directly observe the single-shot spectral dynamics in the initial evolution stage, the zoom-in waterfall plot of the blue soliton's spectral pattern is provided as an example in Fig. 2(b). There are two intriguing features presented in the spectral evolution patterns that mark the collision signature: the complicated dynamic spectral fringes on the main lobe and the rebuilding process of Kelly sidebands with wavelength drifting. Notably, a set of spectral fringes subsequently emerge on the soliton's main lobe. With the perturbed soliton circulating inside the cavity, the density of
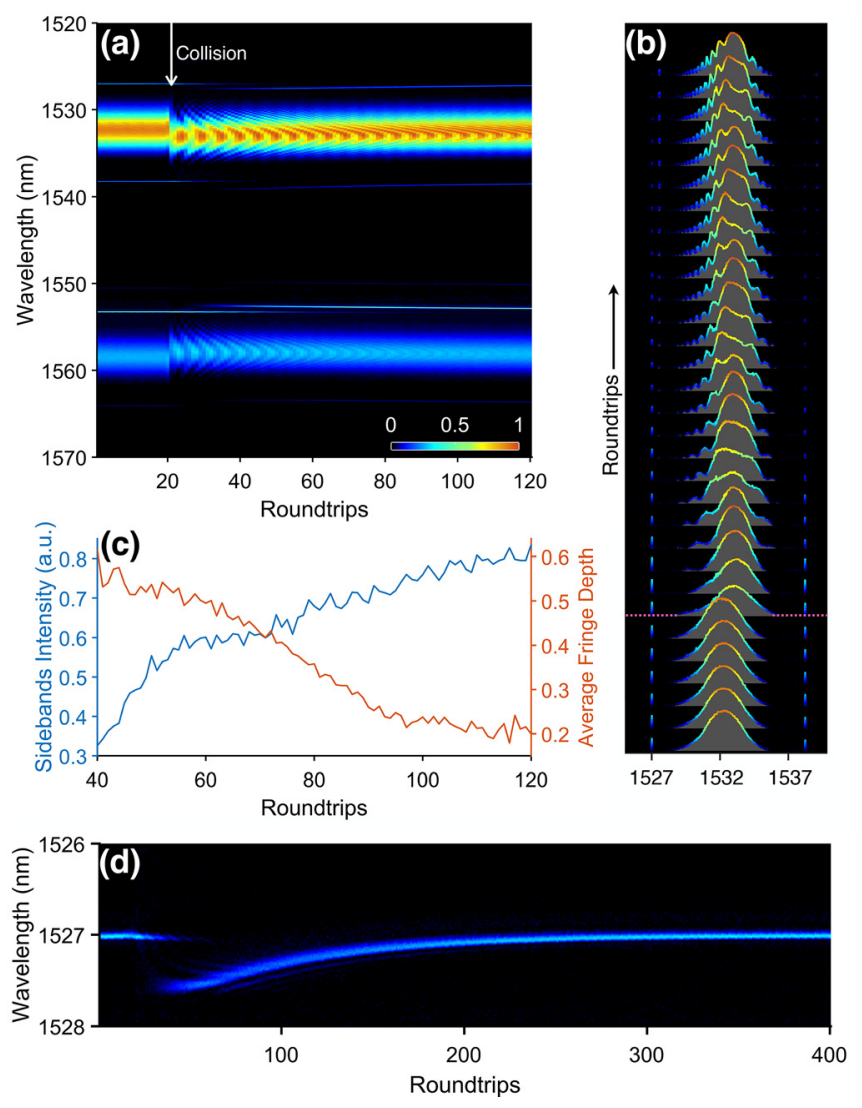

FIG. 2. Collision-induced spectral pattern evolution experimentally measured by single-shot DFT. The collision happens at the 21 st roundtrip. The color bar represents different spectral intensities at certain roundtrips. (a) 2D spectral pattern. (b) The zoom-in waterfall plot of the blue soliton's spectral pattern extracted from the dataset in (a), which contains a set of consecutive single-shot spectra from the 15 th to the 45 th roundtrip. The pink dotted line marks the collision roundtrip. (c) The intensity variation (blue curve) of blue soliton's newly formed sidebands and the average modulation depth variation (orange curve) of blue soliton's spectral fringes during the sideband rebuilding process (from the 40th to the 120th roundtrip) in (a). (d) Zoom-in spectral view of the 1527-nm Kelly sideband's wavelength drifting over 400 roundtrips.

the spectral fringes increases. At the same time, the modulation depth of the fringes declines as the soliton evolves towards its stable state. As another remarkable feature observed simultaneously, the Kelly sidebands of the soliton experience a rebuilding process accompanied by wavelength drifting after the collision. Right after the collision, the sidebands are annihilated in their original wavelengths and subsequently rebuilt at new spectral locations. Although the collision disturbs the Kelly sideband immediately, a few roundtrip-times are required for the newly built sideband to grow large enough to be distinguished from the spectral noise floor. Based on the fact that the intensity of the newly formed sideband surpasses the intensity of the sideband at the original wavelength from the 40th roundtrip, we select the 40th roundtrip as the initial point to perform the quantitative analysis of the post-collision soliton reshaping evolution. Even though the Kelly sideband is not intrinsic to the soliton reshaping process, it is analyzed as a representative feature of the evolution. Here, the intensity variation of blue soliton's newly formed sidebands is provided together with the evolution of the spectral fringe's average modulation depth in Fig. 2(c). It is clearly observed that as the intensity 
of newly formed sidebands increases, the average modulation depth of spectral fringes keeps decaying, which indicates a gradually stabilizing manner of the perturbed soliton. Interestingly, once arising at the new wavelengths, all newly formed Kelly sidebands shift back to their initial wavelengths gradually. To provide a full-range observation of the sideband wavelength drifting, a zoom-in spectral view of the 1527 -nm Kelly sideband over 400 roundtrips is presented in Fig. 2(d) as an example. Finally, the perturbed soliton fully recovers its initially stable mode-locking spectral shapes over hundreds of roundtrips' evolution after the collision.

To gain more insights into the collision-induced spectral dynamics, the soliton interaction length during the collision, which is defined as the co-propagation distance of the two solitons when they remain spatially overlapped, is estimated. Considering the $\sim 4 \mathrm{~nm}$ FWHM bandwidth for both blue and red solitons, and the fact that solitons in the net-anomalous cavity are normally considered as nearly transform-limited, ${ }^{33}$ the approximated FWHM $\operatorname{sech}^{2}$-shaped pulsewidth is about 0.62-ps for the blue soliton and 0.64-ps for the red soliton. Therefore, the interaction length can be approximated as

$$
L_{\text {interact }}=\frac{\Delta t_{1}+\Delta t_{2}}{D \Delta \lambda}=\frac{\Delta t_{1}+\Delta t_{2}}{\left(T_{2}-T_{1}\right) / L},
$$

where $D$ indicates the dispersion of the cavity and $\Delta \lambda$ denotes the wavelength difference between the blue and the red solitons; $\Delta t_{1}$ and $\Delta t_{2}$ are the FWHM pulsewidths of blue and red solitons, respectively; $T_{1}$ and $T_{2}$ are the periods of blue and red solitons, respectively; and $L$ is the cavity length. Under the given parameters, the approximated value of the interaction length is around $3.5 \mathrm{~m}$, which corresponds to an interaction time of $17.1 \mathrm{~ns}$. Since the soliton interaction length during the collision is much shorter than the cavity length, and owing to the cavity inhomogeneity, the strength of collision-induced spectral perturbation is thus location-dependent. We note that the recorded strongest collision-induced spectral dynamics is provided here for the discussion. More significantly, this result illustrates that the mutual interaction between the solitons exists only in the 21st roundtrip, and all the slow spectral evolution afterwards can be regarded as the signature of soliton self-reshaping, which depicts the spectral transition from a disrupted and unsteady state to the final stable mode-locking state.

For further investigation on the experimentally observed laser spectral dynamics, we numerically study the dual-color NPR mode-locked laser and explore the spectral dynamics of the intracavity soliton collision. The simulation models the circulation of optical pulses inside the cavity, which is governed by the coupled Ginzburg-Landau equation $(\mathrm{CGLE})^{1,14,34}$ that portrays the combination of dispersion, Kerr nonlinearity, birefringence, transmission loss, and gain saturation along the fiber. The CGLE is solved by the wellknown split-step Fourier method (SSFM). ${ }^{35,36}$ To facilitate a dual-color-soliton regime, a two-peak gain spectral profile was adopted in the EDF. ${ }^{20}$ Here, we follow the same organization as in the experimental observation (Fig. 2) to present the simulated spectral evolution (Fig. 3). Evidently, the simulated spectral dynamics reveals a remarkable collision signature that reaches good agreement with the experimental
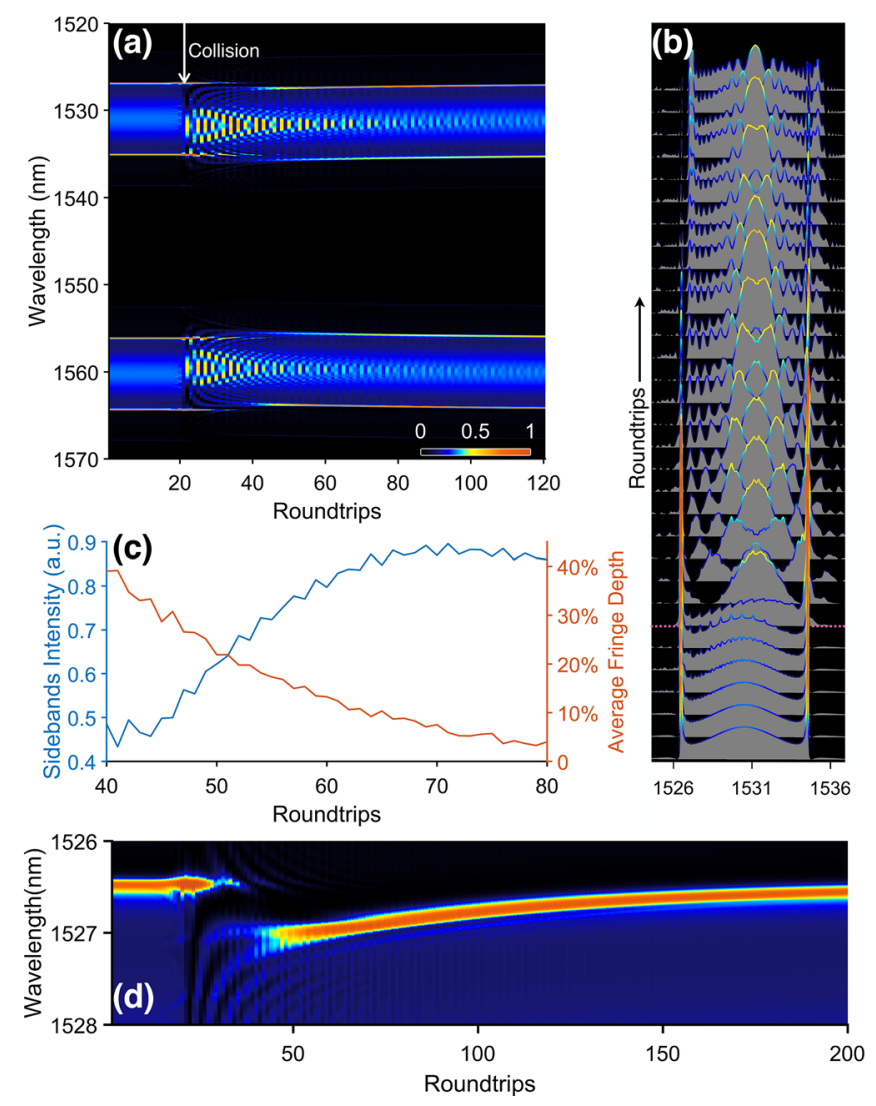

FIG. 3. Collision-induced spectral pattern evolution in the numerical simulation. The collision happens at the 21 st roundtrip. The color bar represents different spectral intensities at certain roundtrips. (a) $2 \mathrm{D}$ spectral pattern. (b) The zoom-in waterfall plot of the blue soliton's spectral pattern extracted from the dataset in (a), which contains a set of consecutive single-shot spectra from the 15 th to the 45 th roundtrip. The pink dotted line marks the collision roundtrip. (c) The intensity variation (blue curve) of blue soliton's newly formed sidebands and the average modulation depth variation (orange curve) of blue soliton's spectral fringes during the sideband rebuilding process (from the 40th to the 80th roundtrip) in (a). (d) Zoom-in spectral view of 1527-nm Kelly sideband's wavelength drifting over 200 roundtrips.

results, while the absence of optical and electrical noise enables a much clearer observation. The minor discrepancies between the simulated and experimentally measured spectral dynamics are mainly attributed to the inevitable noise floor, non-uniform EDFA gain spectrum, and limited detection dynamic range in the experiment. Nevertheless, the qualitative agreement between the simulation and experiment validates our experimentally reconstructed single-shot spectral evolution and proves the accuracy of the simulation model, which enables further analysis of the underlying physical mechanisms behind the intriguing phenomena.

Based on the experimental and simulation results, it is inferred that the observed dynamic spectral evolution is owing to the interference between the optical soliton and the dispersive waves, which are triggered by the cross-phase modulation (XPM) during the soliton collision. It is well known that after suffering from the periodic perturbation caused by the cavity's spatial inhomogeneity (gain, loss, and dispersion) during the circulations, the soliton sheds away a fraction of its energy to form a set of dispersive waves so that it can reshape to its steady state every roundtrip. ${ }^{37-39}$ In our case, the collision induces strong temporal phase perturbations on each soliton through XPM, which triggers 
significant dispersive wave shedding. These dispersive waves possess a similar spectrum with the soliton ${ }^{33,39}$ and will thus interfere with the soliton and cause the modulation fringes on the spectrum. ${ }^{37}$ It is worth noticing that in each single-shot spectrum, the fringe density is lower in the central area and gets higher toward the edge of the soliton spectrum. This feature can be well explained by the chirped nature of the dispersive wave: since the negligible nonlinearity experienced by the dispersive waves is insufficient to balance the dispersion effect, the dispersive waves undergo continuous broadening during circulation, which also explains the growing average spectral fringe density in each consecutive roundtrip during the evolution. On the other hand, the decrease in the modulation depth indicates the decaying of the collision-induced dispersive wave. However, as a special case of the interference, certain wavelengths that satisfy the phase-matching condition experience consistent constructive interference (resonant enhancement) with the soliton in each roundtrip, which leads to the formation of the sharp peak pairs (i.e., Kelly sidebands) symmetrically located at the wings of the soliton spectrum. ${ }^{40-42}$ It is this phase-matching requirement that causes the wavelength drifting of Kelly sidebands during the collision shown in Figs. 2(d) and 3(d). Owing to the strong XPM during soliton collision, the original phase-matching condition is perturbed, which leads to the destruction of sidebands at the original locations. At the same time, new Kelly sidebands at different spectral positions that satisfy the alternated phase-matching requirement start to grow. However, as the soliton reshapes itself back to the stable state, the original phase-matching requirement is gradually restored, which forces the newly formed Kelly sidebands to shift towards their original wavelength positions.

To further verify our analysis on the physical mechanism, the same process is investigated from the time-domain perspective. Given that the dispersive waves are a low intensity wave packet which temporally appear as a wide pedestal of soliton waveform, ${ }^{33,39,43}$ it would be nearly impossible to observe experimentally by using a PD and a real-time oscilloscope. In the limited dynamic range, the significant intensity difference between the soliton waveform and its pedestal prevents the simultaneous observation of soliton and dispersive waves. In addition, the limited experimental temporal resolution $(\sim 50 \mathrm{ps})$ is far not enough to resolve the details. Using the temporal magnification technique with a large dynamic range ${ }^{44}$ to experimentally observe the corresponding temporal dynamics would be of great scientific interest in the future work. Here, the temporal behavior of solitons during the collision-induced evolution is studied via our simulation model (see Fig. 4.). To clearly reveal the dispersive waves, the observation range is restricted around the soliton pedestal, and the temporal intensities outside the upper limit of the observation range are replaced as normalized one. In addition, the two solitons' optical fields are separated from the spectral domain to obtain the individual temporal waveform, which avoids the temporal overlapping of pedestal at the collision point.

Right after the collision, noticeable dispersive wave shedding subsequently appears on the soliton's waveform pedestal as shown in Fig. 4(c), as they symmetrically spread

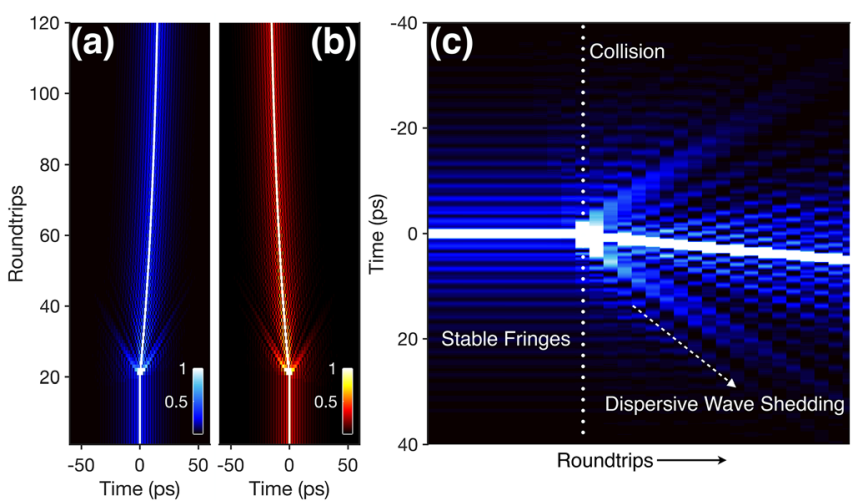

FIG. 4. The simulated collision-induced consecutive temporal dynamics. The collision happens at the 21 st roundtrip. The color bar represents different temporal intensities at certain roundtrips. (a) Blue soliton. (b) Red soliton. (c) Zoom-in temporal dynamics of the blue soliton extracted from the dataset in (a), from the 10th to the 40th roundtrip. The white dotted line marks the collision roundtrip.

out from the main lobe (denoted by the central white line). This concretely verifies our interpretation on the increased spectral fringe density in both experiment [see Fig. 2(b)] and simulation [see Fig. 3(b)], as larger chirping of the dispersive wave leads to a denser spectral interference pattern. Meanwhile, the soliton main lobe undergoes a temporal drifting, which indicates a slight change in the soliton's group velocity induced by the XPM during soliton collision. Specifically, the group velocity difference between two solitons becomes smaller right after the collision, which can be observed from the tilting of the temporal evolution trace in Figs. 4(a) and 4(b). This matches well with the phenomenon that the optical spectra of both solitons shift towards each other right after the collision in Figs. 2(a) and 3(a) and can be intuitively understood as a soliton trapping effect. ${ }^{45}$ In addition, the stable temporal fringes beside the main lobe in Fig. 4(c) correspond to the temporal beating between the symmetrically located Kelly sidebands. Therefore, the temporal dynamics further confirm our interpretation on the experimentally observed phenomena and the explanation of the soliton-collision mechanism.

In conclusion, we have experimentally observed the round-trip resolved spectral dynamics of intracavity dualcolor-soliton collision in a mode-locked fiber laser by dispersive Fourier transform. Numerical simulation is performed to verify our experimental observation and offer a temporal perspective to further confirm our interpretation on the collision-induced dispersive wave shedding and the dynamic soliton-dispersive wave interference during the soliton reshaping process. Our finding not only serves as a demonstration of solitons' unique capability of self-stabilization, but also offers an insight into analyzing the intricate dynamic interaction of solitons in dissipative systems.

The authors acknowledge the fruitful discussion with Dr. Shu-Wei Huang from the University of Colorado, Boulder, on the soliton dynamics. This work was partially supported by the grants from the Research Grants Council of the Hong Kong Special Administrative Region, China (Project Nos. HKU 17208414, HKU 17205215, CityU T42103/16-N, and E-HKU701/17), the National Natural Science 
Foundation of China (No. N_HKU712/16), and GD-HK Technology Cooperation Funding Scheme (No. GHP/050/ 14GD).

${ }^{1}$ G. P. Agrawal, Nonlinear Fiber Optics (Academic Press, 2013).

${ }^{2}$ P. Grelu and N. Akhmediev, Nat. Photonics 6, 84 (2012).

${ }^{3}$ B. Li, S.-W. Huang, Y. Li, C. W. Wong, and K. K. Y. Wong, Nat. Commun. 8, 61 (2017).

${ }^{4}$ B. Li, Y. Yu, X. Wei, Y. Xu, K. K. Tsia, and K. K. Y. Wong, Opt. Express 25, 8751 (2017).

${ }^{5}$ Y. Yu, B. Li, X. Wei, Y. Xu, K. K. Tsia, and K. K. Y. Wong, Appl. Phys. Lett. 110, 201107 (2017).

${ }^{6}$ X. Wei, Y. Xu, and K. K. Y. Wong, Opt. Lett. 40, 3005 (2015).

${ }^{7}$ K. Krupa, K. Nithyanandan, and P. Grelu, Optica 4, 1239 (2017).

${ }^{8}$ M. Liu, Z.-R. Cai, S. Hu, A.-P. Luo, C.-J. Zhao, H. Zhang, W.-C. Xu, and Z.-C. Luo, Opt. Lett. 40, 4767 (2015).

${ }^{9}$ C. Lecaplain, P. Grelu, J. M. Soto-Crespo, and N. Akhmediev, Phys. Rev. Lett. 108, 233901 (2012).

${ }^{10}$ J. M. Dudley, F. Dias, M. Erkintalo, and G. Genty, Nat. Photonics 8, 755 (2014).

${ }^{11}$ A. F. Runge, N. G. Broderick, and M. Erkintalo, Optica 2, 36 (2015).

${ }^{12}$ S. T. Cundiff, J. M. Soto-Crespo, and N. Akhmediev, Phys. Rev. Lett. 88, 073903 (2002)

${ }^{13}$ M. Liu, A.-P. Luo, Y.-R. Yan, S. Hu, Y.-C. Liu, H. Cui, Z.-C. Luo, and W.-C. Xu, Opt. Lett. 41, 1181 (2016).

${ }^{14}$ D. Tang, L.-M. Zhao, B. Zhao, and A. Liu, Phys. Rev. A 72, 043816 (2005).

${ }^{15}$ M. Stratmann, T. Pagel, and F. Mitschke, Phys. Rev. Lett. 95, 143902 (2005).

${ }^{16}$ G. Herink, F. Kurtz, B. Jalali, D. Solli, and C. Ropers, Science 356, 50 (2017).

${ }^{17}$ S. Chouli and P. Grelu, Phys. Rev. A 81, 063829 (2010).

${ }^{18}$ V. J. Matsas, T. P. Newson, D. J. Richardson, and D. N. Payne, Electron. Lett. 28, 1391 (1992).

${ }^{19}$ X. Zhao, Z. Zheng, L. Liu, Y. Liu, Y. Jiang, X. Yang, and J. Zhu, Opt. Express 19, 1168 (2011).

${ }^{20}$ D. Mao and H. Lu, J. Opt. Soc. Am. B 29, 2819 (2012).

${ }^{21}$ R. Liao, Y. Song, L. Chai, and M.-L. Hu, in Conference on Lasers and Electro-Optics, OSA Technical Digest (Optical Society of America, 2017), p. SM4L.5.
${ }^{22}$ X. Liu, D. Han, Z. Sun, C. Zeng, H. Lu, D. Mao, Y. Cui, and F. Wang, Sci. Rep. 3, 2718 (2013).

${ }^{23}$ Y. Wang, J. Li, B. Zhai, Y. Hu, K. Mo, R. Lu, and Y. Liu, Opt. Express 24, 15299 (2016)

${ }^{24}$ I. Coddington, N. Newbury, and W. Swann, Optica 3, 414 (2016).

${ }^{25} \mathrm{G}$. Hu, T. Li, Y. Pan, X. Zhao, M. Zhang, and Z. Zheng, in CLEO: QELS Fundamental Science (Optical Society of America, 2017), p. JTh2A.134.

${ }^{26}$ X. Zhao, G. Hu, B. Zhao, C. Li, Y. Pan, Y. Liu, T. Yasui, and Z. Zheng, Opt. Express 24, 21833 (2016).

${ }^{27}$ J. Chen, R. Wang, T. Li, C. Li, Y. Pan, X. Zhao, J. Liu, and Z. Zheng, in CLEO: Applications and Technology (Optical Society of America, 2017), p. JW2A.5.

${ }^{28}$ Y. Liu, X. Zhao, G. Hu, C. Li, B. Zhao, and Z. Zheng, Opt. Express 24, 21392 (2016).

${ }^{29}$ A. Mahjoubfar, D. V. Churkin, S. Barland, N. Broderick, S. K. Turitsyn, and B. Jalali, Nat. Photonics 11, 341 (2017).

${ }^{30}$ K. Goda and B. Jalali, Nat. Photonics 7, 102 (2013).

${ }^{31}$ R. Woodward and E. Kelleher, Phys. Rev. E 93, 032221 (2016).

${ }^{32}$ K. Goda, D. R. Solli, K. K. Tsia, and B. Jalali, Phys. Rev. A 80, 043821 (2009).

${ }^{33}$ L. E. Nelson, D. J. Jones, K. Tamura, H. A. Haus, and E. P. Ippen, Appl. Phys. B 65, 277 (1997).

${ }^{34}$ D. Y. Tang, L. M. Zhao, and B. Zhao, Opt. Express 13, 2289 (2005).

${ }^{35}$ J. Weideman and B. Herbst, SIAM J. Numer. Anal. 23, 485 (1986).

${ }^{36}$ O. V. Sinkin, R. Holzlöhner, J. Zweck, and C. R. Menyuk, J. Lightwave Technol. 21, 61 (2003).

${ }^{37}$ M. W. Chbat, P. R. Prucnal, M. N. Islam, C. E. Soccolich, and J. P. Gordon, J. Opt. Soc. Am. B 10, 1386 (1993).

${ }^{38}$ C. Desem and P. Chu, Opt. Lett. 11, 248 (1986).

${ }^{39}$ J. P. Gordon, J. Opt. Soc. Am. B 9, 91 (1992).

${ }^{40}$ M. L. Dennis and I. N. Duling, IEEE J. Quantum Electron. 30, 1469 (1994).

${ }^{41}$ S. M. J. Kelly, Electron. Lett. 28, 806 (1992).

${ }^{42}$ N. J. Smith, K. Blow, and I. Andonovic, J. Lightwave Technol. 10, 1329 (1992).

${ }^{43}$ R. Weill, A. Bekker, V. Smulakovsky, B. Fischer, and O. Gat, Phys. Rev. A 83, 043831 (2011).

${ }^{44}$ P. Suret, R. E. Koussaifi, A. Tikan, C. Evain, S. Randoux, C. Szwaj, and S. Bielawski, Nat. Commun. 7, 13136 (2016).

${ }^{45}$ M. N. Islam, C. D. Poole, and J. P. Gordon, Opt. Lett. 14, 1011 (1989). 